\title{
La ruptura de las uniones en Colombia y sus diferenciales. Una aproximación biográfica a partir de la Encuesta Nacional de Demografía y Salud de 2015*
}

DOI: https://doi.org/10.18046/recs.i33.4058

\author{
Union Dissolution in Colombia and their Determinants. \\ A Biographical Approach Based on the 2015 Demographics \\ and Health Survey
}

\author{
Fernando Ruiz-Vallejo**
}

Asociación Profamilia (Bogotá, Colombia)

\footnotetext{
* El presente trabajo es una adaptación de un capítulo de la tesis del autor, en el marco del Doctorado en Demografía del Centro de Estudios Demográficos (CED) de la Universidad Autónoma de Barcelona, bajo la dirección de los profesores Albert Esteve y Montserrat Solsona. El autor agradece a Diederik Boertien, investigador del CED, por la orientación académica a lo largo del doctorado; y también a COLCIENCIAS por la beca otorgada para realizar el doctorado, a través de su convocatoria núm. 617 de 2013. Artículo de investigación recibido el 04.06.2020 y aceptado el 03.01.2021.

** Sociólogo de la Universidad Nacional de Colombia, Maestro en Población y Desarrollo por la Facultad Latinoamericana de Ciencias Sociales, sede México, y Doctor en Demografía por el Centro de Estudios Demográficos de la Universidad Autónoma de Barcelona (España). Ha realizado investigaciones sobre violencia de pareja, derechos sexuales y reproductivos; salud y población; migración internacional y trata de personas. Sus trabajos tienen un enfoque de género, curso de vida y desigualdades sociales. Correo electrónico: fernando.ruiz@profamilia.org.co ORCID: https://orcid. org/0000-0003-1121-2562
} 


\section{Cómo citar/How to cite}

Ruiz-Vallejo, Fernando (2021). La ruptura de las uniones en Colombia y sus diferenciales.

Una aproximación biográfica a partir de la Encuesta Nacional de Demografía y Salud de 2015.

Revista CS, 33, 103-139. https://doi.org/10.18046/recs.i33.4058 
Resumen

Abstract

El presente trabajo ofrece una estimación de la intensidad y el calendario de la primera disolución de la unión en Colombia y sus departamentos, a partir de la aplicación de técnicas de análisis de supervivencia con información de la Encuesta Nacional de Demografía y Salud de $2015(\mathrm{n}=24,823)$. Los resultados indican que, después de 20 años, el $48 \%$ de las uniones se disuelven. El mayor riesgo de separación se asocia con las cohortes más jóvenes, la unión libre, la urbanidad, la alta escolaridad, los calendarios tempranos de unión y las brechas de edad en donde la mujer tiene una edad similar o es mayor que su pareja. Por el contrario, los hijos e hijas en edades preescolares reducen dicho riesgo.

PALABRAS CLAVE:

divorcio, separación, diferenciales de la disolución de las uniones, Colombia

This study estimates the intensity and timing of the first union dissolution in Colombia and its regions, based on the application of survival analysis techniques with information from the 2015 Demographics and Health Survey $(n=24,823)$. The results demonstrate that after 20 years, $48 \%$ of unions were dissolved. The highest risk is associated with younger cohorts, common law marriage, early age at union, and age gaps where the woman is similar in age or older than her partner. Similarly, a higher level of education, as well as living in urban areas or certain regions, increases the risk of union dissolution. On the contrary, having preschool children was identified as a characteristic reducing this risk.

\section{KEYWORDS:}

Divorce, Union Dissolution, Union Dissolution Predictors, Colombia 



\section{Introducción}

La ruptura de las uniones ${ }^{1}$ ha sido un tema de constante preocupación en los medios de comunicación nacionales. El 29 de agosto de 2018, a raíz de las declaraciones del superintendente de Notariado y Registro sobre el aumento de los divorcios, el portal web de una emisora de amplia difusión iniciaba su publicación con la siguiente pregunta: “¿El divorcio está de moda en Colombia?" (Espejo, 2018). La respuesta positiva del superintendente indicaba que, en efecto, los divorcios han venido en aumento, hasta llegar a un nivel de 3,5 matrimonios por cada divorcio; siendo Bogotá, Cali y Medellín las ciudades con las tasas más altas. Sin embargo, el funcionario también afirmaba que en lugares como Puerto Carreño no se habían celebrado nuevas nupcias y, en cambio, se habían tramitado 4 divorcios. De esta manera, se advierte la limitación de esta fuente, al estar supeditada, por una parte, a la ocurrencia de dos eventos (matrimonios y divorcios) de distintas personas en el mismo año y, por otra, a la intervención del Estado en la formación y disolución de las uniones.

Los datos de la Superintendencia contrastan con el bajo porcentaje de disoluciones reportado por los censos de población entre 1951 y 2005, los cuales muestran que, entre las mujeres de 30 a 34 años, dicha cifra nunca fue superior al $8 \%$ (Ruiz-Vallejo, 2020: 170). Recientemente, el censo de 2018 estimó este valor en $9 \%$ y, a partir de la pregunta por el estado civil actual, permitió distinguir el tipo particular de disolución. De esta manera, se observa que la separación de una unión libre contribuyó con el $77 \%$ del estado civil separada o divorciada, seguida de la separación de un matrimonio con $17 \%$, y tan solo $6 \%$ correspondía a un divorcio formal (Departamento Administrativo Nacional de Estadística [DANE], 2020). Este último dato permite visualizar el alto nivel de subregistro de los datos de la Superintendencia, dado que la mayor parte de las disoluciones provienen de las uniones libres. Por tanto, cualquier estimación que no tenga en cuenta este tipo de unión conlleva un alto grado de omisión.

Por otra parte, aunque el censo y otras encuestas incluyen las uniones libres, no tienen en cuenta el orden de la unión declarado en el estado civil actual, es decir, no permiten identificar las experiencias pasadas de disolución de una persona que, en el momento de responder las preguntas, se encontraba casada o en unión libre (Flórez; Sánchez, 2013). Ante esta situación, la medición de la ruptura de las uniones queda supeditada a encuestas probabilísticas que, por un lado, recopilen las fechas de inicio y finalización de las uniones experimentadas por un individuo a lo largo de

1. A lo largo del texto se usan los términos disolución, ruptura y separación de manera alternada, para hacer referencia a la finalización de la primera unión, independientemente de la etapa legal en que se encuentre la separación. 
su vida (denominadas historias de unión), y por otro lado, que incluyan los arreglos de pareja informales que eventualmente no dejarán constancia en los registros oficiales (Cabella, 2008; Goldman, 1981; Ojeda, 1986; Pérez-Amador; Ojeda, 2016).

En el caso particular de Colombia, no existen estudios posteriores a la década de los ochenta, que, a través de métodos biográficos, estimen a nivel nacional y regional las rupturas de las uniones. El último año de referencia es 1985, cuando se realizó la encuesta urbana de Zamudio y Rubiano (1991), a partir de la cual se actualizaron las estimaciones basadas en la Encuesta Mundial de Fecundidad (EMF) de 1976 (Goldman, 1981). Desde entonces, han pasado más de 30 años en los cuales el país ha experimentados cambios sociales y demográficos importantes, con repercusiones en los niveles actuales de disolución conyugal.

Recuperando la pregunta formulada al inicio de la introducción, el presente artículo se plantea tres inquietudes en torno a la preocupación mediática de la moda del divorcio: ¿cuál es el nivel nacional y departamental de separación conyugal, teniendo en cuenta tanto los matrimonios como las uniones libres?, ¿han aumentado en el tiempo?, y ¿̇cuáles son las características de la unión, los individuos y los contextos, con mayores probabilidades de ruptura conyugal?

Para responder dichos interrogantes, el presente artículo da continuidad a las investigaciones demográficas de corte biográfico realizadas sobre la separación en Colombia (Goldman, 1981; Zamudio; Rubiano, 1991), definiendo tres objetivos específicos: 1) medir el nivel nacional y departamental de disolución de la primera unión en distintas duraciones (5, 10, 15 y 20 años de haberse iniciado la unión), 2) comparar la duración de las uniones (matrimonios vs. uniones libres) de tres cohortes para establecer si las uniones contemporáneas son más cortas que las antiguas, y 3) identificar los diferenciales asociados a la disolución de la primera unión, de acuerdo con tres tipos de variables: las características de la unión (cohorte y tipo de arreglo conyugal), del individuo y su pareja (edad a la unión, diferencia de edad con la pareja, escolaridad, hijos previos a la unión, número de hijos y edad de los mismos durante la unión), y del contexto de residencia actual (zona y región).

Es importante advertir que, ante las restricciones de la fuente empleada para el presente trabajo, el alcance del mismo es meramente descriptivo y no explicativo. Su principal contribución es ofrecer mediciones empíricas que permitan cuantificar los niveles y diferenciales asociados a la ruptura de la primera unión en el país, más que verificar grandes hipótesis sobre el cambio social vinculado con este fenómeno.

Además de la presente introducción, el artículo contiene cinco secciones. La primera ofrece una contextualización breve de los cambios demográficos y sociales de los últimos 40 años, en los cuales se enmarca la evolución de las rupturas de las uniones. La segunda sección recopila los principales antecedentes de la literatura 
demográfica alrededor de los diferenciales de la separación, con especial interés en los estudios latinoamericanos. El tercer apartado corresponde a la descripción de la metodología y las técnicas empleadas, incluyendo la definición de la población y la construcción de las variables de análisis. Los resultados se incluyen en el cuarto apartado, organizados en tres momentos: la descripción de las tres cohortes de unión a partir de las variables independientes, la estimación de las duraciones de las uniones a nivel nacional y para 33 unidades territoriales, y los resultados de los modelos de regresión que identifican los diferenciales de las rupturas. Finalmente, en la quinta y última sección, se presentan las conclusiones, destacando los principales hallazgos en diálogo con la literatura y se señalan las líneas futuras de investigación.

\section{Los cambios demográficos y sociales relacionados con las rupturas de las uniones}

La disolución de las uniones es un fenómeno demográfico que permite observar los cambios sociales vinculados principalmente con la mayor participación de las mujeres en la esfera pública (Cherlin, 2010; Esping-Andersen; Billari, 2015; Goldscheider; Bernhardt; Lappegård, 2015) y con la consolidación de tres procesos contemporáneos fundamentales para los cambios familiares: la individualización, la dislocación progresiva de la triada conyugalidad-sexualidad-reproducción, y la trasformación de los valores que sustentan el cambio demográfico (Beck-Gernsheim, 2003; Giddens, 1997; Lesthaeghe, 2010; Van de Kaa, 2002).

Los ajustes en los regímenes demográficos como consecuencia del descenso de la fecundidad, el aumento en la esperanza de vida y las dinámicas migratorias han incidido de manera significativa en la estructura, organización y funcionamiento de las familias (Ariza; Oliveira, 2001; García; Rojas, 2004); sin embargo, estos cambios se dan de distintas formas y con intensidades heterogéneas. Entre las particularidades del caso latinoamericano, parece existir un consenso sobre la coexistencia de dos modelos de familia. Por un lado, se identifica un patrón nuevo en el que hay mayor autonomía de las mujeres, un alto nivel de control de la reproducción y menos desigualdad en la distribución de las tareas del hogar; y por otro, se constata la persistencia de un modelo tradicional en el cual se mantienen la dependencia subjetiva de las mujeres y la división sexual de los roles de género (Fernández, 2010). De esta manera, la configuración de tales regímenes demográficos podría derivar en distintos comportamientos de la disolución de las uniones.

En el caso particular de Colombia, aunque son limitadas las estimaciones mediante métodos biográficos, algunos estudios poblacionales han confirmado el 
aumento progresivo de la ruptura de las uniones desde la década de los setenta. En ese sentido, destacan los trabajos de Carmen Elisa Flórez, quien a través de fuentes transversales, ha constatado el incremento de este fenómeno, tanto en las principales ciudades (Flórez, 2004) como desde una mirada conjunta del país y sus regiones (Flórez; Sánchez, 2013). Asimismo, mediante la estimación de métodos indirectos, otro estudio encontró una tendencia similar, al afirmar que, entre las mujeres de 30 a 34 años, el porcentaje de las que habían experimentado al menos una ruptura fue de $34 \%$ en 2015, contrastando con el $17 \%$ reportado para 1976 (Ruiz-Vallejo, 2020:173).

La segunda mitad del siglo XX significó una transformación social importante para Colombia, caracterizada, entre otras cosas, por un aumento sustantivo del nivel educativo, especialmente de las mujeres, lo cual contribuyó a su incorporación en el mercado laboral y a la reducción sustantiva de la fecundidad, generando así un cambio favorable en las actitudes y valores vinculados al menor tamaño de las familias, el rol de los hijos y de las mujeres en el hogar (Flórez, 200o). Estas tendencias han continuado en el siglo XXI, configurando así el escenario actual para comprender los patrones de disolución de las uniones. La Figura 1 resume dicho escenario en términos de cuatro variables fundamentales del contexto de las rupturas entre 1986 y 2015: el aumento de la escolaridad, la participación laboral de las mujeres, el incremento de la unión libre y la reducción de la fecundidad. Adicionalmente, incluye los momentos claves en el marco legal que ha regulado la formación y disolución de las uniones en el país (indicadas con líneas discontinuas en la Figura 1).

Mientras en 1986, 30 \% de las mujeres del grupo de edad 25-29 años declaraba estar trabajando en ese momento, en 2015 este valor fue de $63 \%$. En ese mismo período, la unión libre, en el mismo grupo de edad, pasó del $31 \%$ de todas las uniones, a representar el $70 \%$. Este incremento ha sido aún más sorprendente en el acceso de las mujeres a la educación superior. Tomando como referencia el grupo 30-49 años, se observa que tan solo $3 \%$ de las mujeres contaban con estudios de educación superior en 1986; en 2015, este porcentaje alcanzó un $23 \%$, es decir, 6,2 veces más alto (Corporación Centro Regional de Población; Ministerio de Salud de Colombia; Institute for Resource Development, 1988; Profamilia; Ministerio de Salud y Protección Social, 2017).

Estos patrones se producen en el contexto de las modificaciones de los marcos jurídicos que regulan la formación y la disolución de las uniones. La aprobación del divorcio vincular (la disolución legal del matrimonio civil) en 1976 representó un momento importante en el reconocimiento de la voluntad individual para concluir legalmente relaciones insatisfactorias que anteriormente no se podían disolver. Este derecho se extendió a los matrimonios religiosos en 1992, con la modificación del Código Civil, mediante el cual se garantizaba el poder del Estado para disolver este 
FIGURA 1 Elementos del contexto social, reproductivo y jurídico de la ruptura de las uniones en Colombia, 1976-2016

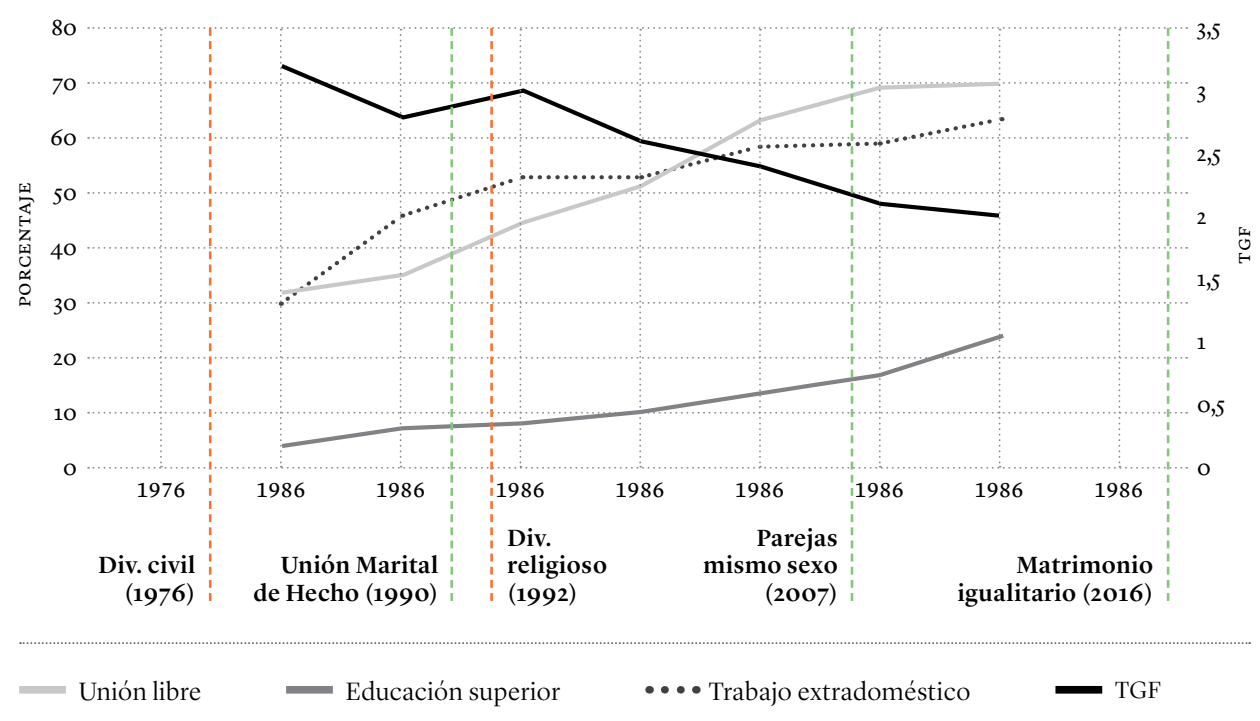

Nota: el porcentaje de unión libre y de trabajo extradoméstico se estima para el grupo de edad 25-29 años; el de educación superior, para el grupo de 30-49 años; y la Tasa Global de Fecundidad, para el grupo 15-49 años. Todos los valores corresponden a las mujeres.

Fuente: cálculos propios a partir de ocho Demographic and Health Survey (DHS), 1986-2016. Los datos del contexto jurídico se obtuvieron a partir de Torrado (2016) y Colombia Diversa (2017).

tipo de uniones. En cuanto a la regulación legal de las uniones, a pesar de la importancia numérica de la unión libre, Colombia solo las reconoció formalmente en 1990, mediante la figura de la unión marital de hecho, aunque únicamente para las parejas heterosexuales. En 2007, se modificó nuevamente la ley para incluir a las parejas del mismo sexo, y en 2016, finalmente se aprobó el matrimonio igualitario mediante los desarrollos jurisprudenciales de la Corte Constitucional (Colombia Diversa, 2017).

\section{Diferenciales de la ruptura de las uniones en América Latina y resultados esperados para Colombia}

Al igual que en el país, los estudios sobre América Latina han confirmado el aumento de la disolución de las uniones en Argentina (Santillán; Street, 2005), Brasil (Miranda-Ribeiro, 1993), Chile (Salinas-Ulloa, 2018), México (Gómez, 2011; Ojeda; 
González, 2008; Pérez-Amador, 2008; Pérez-Amador; Ojeda, 2016; Solís; Ferraris, 2014; Solís; Puga, 2009) y Uruguay (Bucheli; Vigna, 2005; Cabella, 2010). Las variaciones en la intensidad y calendario del fenómeno responden a diferentes aspectos relacionados con las características de la unión, los individuos involucrados en ella, la presencia de hijos y, finalmente, las características de los contextos inmediatos.

La evidencia disponible sobre estos predictores, factores o diferenciales es heterogénea, no solo en función del país analizado, sino también del momento en el que se realiza la investigación. Por ello, en el presente apartado se recopilarán los hallazgos empíricos identificados en la literatura sociodemográfica de los países latinoamericanos ${ }^{2}$, e inmediatamente después, se formulará un enunciado sobre los resultados que podrían esperarse para el caso colombiano, y que serán materia de análisis de este estudio. Al respecto, vale la pena mencionar el uso intencional de la expresión resultado esperado, a cambio de hipótesis, dado el alcance descriptivo de nuestro abordaje, el cual no pretende comprobar o refutar alguna teoría explicativa sobre la separación conyugal, sino que, tal como fue mencionado en la introducción, busca cuantificar el nivel de disolución e identificar los diferenciales asociados.

\section{Características de la unión}

El incremento de las rupturas se confirmaría si las personas más jóvenes disuelven en mayor medida sus uniones en comparación con los grupos de edad mayores (cohorte de nacimiento), o si las uniones más recientes tienen una duración más corta que las formadas mucho antes, es decir, de acuerdo a su cohorte de unión (Lyngstad; Jalovaara, 2010). Del primero de estos abordajes metodológicos provienen los estudios que constatan dicho aumento en México (Gómez, 2011; Ojeda; González, 2008; Pérez-Amador, 2008; Pérez-Amador; Ojeda, 2016; Solís; Ferraris, 2014; Solís; Puga, 2009), Uruguay (Bucheli; Vigna, 2005; Cabella, 2010), y en menor medida Brasil (Miranda-Ribeiro, 1993), Argentina (Santillán; Street, 2005) y Chile (Salinas-Ulloa, 2018). Cuando se toma la cohorte de unión como referencia, la evidencia indica una menor duración de las uniones contemporáneas en el caso de Argentina (Santillán; Street, 2005), México (Solís; Ferraris, 2014) y Uruguay (Cabella, 2008). De esta manera en el presente estudio se espera encontrar un aumento de la ruptura de

2. El presente artículo no se propone documentar la amplia diversidad de diferenciales. Únicamente se incluyen aquellos para los cuales se dispone información en la fuente consultada aquí y que fueron documentados ampliamente en otro trabajo (Ruiz-Vallejo, 2018). Para una revisión de la literatura de Europa y Estados Unidos, se recomienda consultar revisiones especializadas (Amato, 2000; 2010; Mortelmans, 2020; Raley; Sweeney, 2020). 
las uniones conformadas a partir de 2004, en comparación con las cohortes anteriores (resultado esperado núm. 1 o RE1 en adelante).

La mayor fragilidad de las uniones contemporáneas puede obedecer a múltiples razones, siendo una de ellas el cambio en la composición de los arreglos conyugales, dado por el aumento de la unión libre en todos los niveles educativos y especialmente entre los grupos de mayor escolaridad, observándose su coexistencia con el matrimonio (Esteve; Lesthaeghe; Lopez-Gay, 2012). Ante este rasgo dual del sistema de nupcialidad latinoamericano (Castro-Martín, 2002), también hay evidencia que confirma el mayor nivel de disolución de las uniones libres tanto en la región (Cabella, 2010; Miranda-Ribeiro, 1993; Ojeda, 1986; Pérez-Amador; Ojeda, 2016; Santillán; Street, 2005; Solís; Ferraris, 2014) como en el caso particular de Colombia (Goldman, 1981; Zamudio; Rubiano, 1991). Bajo este escenario se inscribe el segundo resultado esperado, proponiendo que, ante la disminución de los matrimonios como arreglo conyugal, estos tendrán una duración mayor en comparación con las uniones libres (RE2), es decir, las personas que optan por el matrimonio en la actualidad, podrían constituir un grupo con características muy particulares en términos de sus valores, percepciones sobre el compromiso y condiciones materiales específicas (Jalovaara; Kulu, 2018), que repercutirán en uniones más duraderas.

\section{Características individuales y de la pareja}

La mayor presión económica de las parejas jóvenes, así como su posible inexperiencia en el manejo de las tensiones conyugales, puede generar un riesgo mayor de ruptura en comparación con quienes inician sus relaciones más tarde (Kuperberg, 2014). Sin embargo, se ha discutido el carácter lineal de dicho efecto, dado que, ante una eventual presión por tener hijos, las mujeres al final de su período reproductivo podrían flexibilizar sus opciones de pareja, conllevándolas a formar uniones con individuos menos compatibles, lo cual a su vez incrementa el riesgo de ruptura (Lehrer, 2008). En Latinoamérica no se ha encontrado evidencia de esta última situación, pero sí de la primera, es decir, del mayor riesgo reportado por las mujeres que establecen su primera unión a edades tempranas (Bucheli; Vigna, 2005; Cabella, 2010; Goldman, 1981; Miranda-Ribeiro, 1993; Ojeda, 1986; Ojeda; González, 20o8; Pérez-Amador; Ojeda, 2016; Santillán; Street, 2005; Solís; Ferraris, 2014; Zamudio; Rubiano, 1991). De esta manera, y considerando los pocos cambios de la edad a la primera unión en Colombia (Flórez; Sánchez, 2013), se espera que aún hoy cada año de edad en que se postergue la unión reducirá el riesgo de disolución ( $\left.\mathrm{RE}_{3}\right)$.

Por otra parte, la perspectiva relacional es fundamental para comprender el rol de la edad en la disolución. No solo unirse en edades jóvenes es un elemento im- 
portante, sino también la edad de la pareja y su correspondiente brecha etaria, en lo que se conoce como la hipótesis de la homogamia. Desde este ángulo, las parejas más similares en términos de su escolaridad, raza/etnia, tradiciones, actitudes, culturas y edad son también las más duraderas (Kalmijn, 1998). Esto se confirma en el caso de la homogamia educativa, en donde Latinoamérica reporta altos niveles (Esteve, López; McCaa, 2013; San Juan; Esteve, 2019), pero que no se corresponde con la homogamia de edad, en donde las brechas entre las parejas es aún amplia. Bajo este escenario, en Brasil y Colombia se identifica un patrón conyugal de un hombre unido con una mujer más joven que, contrario a la hipótesis de la homogamia, exhibe un menor riesgo de disolución en comparación con las parejas más igualitarias en su edad (Zamudio; Rubiano, 1991; Miranda-Ribeiro, 1993). De esta manera, la presente investigación espera encontrar una tendencia similar, es decir, un menor riesgo de ruptura de las parejas en donde el hombre es mayor que la mujer (RE4).

Cuando el nivel educativo se toma como una característica individual, los trabajos pioneros de William Goode (1962) proponían que, en una etapa inicial de divorciali$d a d$, las mujeres con alta escolaridad serían aquellas con la mayor probabilidad de disolver sus uniones, por contar con más recursos para afrontar las sanciones sociales y los desajustes en la vida económica familiar. Posteriormente, las sociedades tenderían a aceptar el divorcio mediante la regulación de las rupturas y la normalización cultural del mismo, a tal punto que se extendería a todos los segmentos sociales, diluyendo así el carácter diferenciador de la educación. De hecho, actualmente, en los países del norte de Europa, esta relación ha empezado a invertirse, siendo ahora las mujeres con mayor nivel educativo las que reportan las tasas de disolución más bajas (Härkönen; Dronkers, 2006).

Dadas las dinámicas del orden sexual colombiano, la investigación ha mostrado un patrón contrario al escenario europeo. En la década del setenta se identificó a las mujeres con menor escolaridad como aquellas con la mayor proporción de uniones disueltas (Goldman, 1981), posiblemente por situaciones de abandono que no eran captadas por el instrumento usado en el estudio de Goldman. En efecto, el trabajo de Zamudio y Rubiano (1991) mostraba cómo, a mediados de los ochenta, la relación entre escolaridad y separación dibujaba una $U$ con la mayor proporción de uniones disueltas por parte de las mujeres con mayor y menor nivel educativo. Asimismo, en este trabajo se comprobó que fueron los hombres quienes mayoritariamente tomaron la iniciativa de disolver la unión, contrario a lo ocurrido en Europa, donde en mayor medida son las mujeres las que inician el proceso (Härkönen, 2014).

En la actualidad, los países latinoamericanos muestran patrones diferentes en el rol de la educación como diferencial de la separación conyugal. Por un lado, países como Brasil, Chile y México estarían en una etapa inicial de divorcialidad con 
mayores riesgos de ruptura para las mujeres con alta escolaridad (Miranda-Ribeiro, 1993; Pérez-Amador, 2008; Salinas-Ulloa, 2018), aunque hay que destacar que en el caso particular de México la tendencia empieza a revertirse según los últimos datos disponibles (Pérez-Amador; Ojeda, 2016). Por otro lado, se encontrarían países como Uruguay, en donde la educación ya no representa un diferencial de la disolución (Cabella, 2010). A partir de la evidencia disponible, proponemos como resultado esperado núm. 5 (RE5) que las mujeres con mayor escolaridad son quienes tienen más riesgo de disolver sus uniones.

Los últimos diferenciales incluidos en el grupo de características individuales y de la pareja son haber tenido hijos antes de iniciar la unión y el número de hijos durante la unión, así como la edad de los mismos. La literatura internacional ha encontrado que haber tenido hijos previos a la unión incrementa las probabilidades de divorciarse (Ermisch, 2003; Waite; Lillard, 1991). En Latinoamericana, este efecto también se ha confirmado en la mayor parte de los países con información disponible (Goldman, 1981; Miranda-Ribeiro, 1993; Ojeda, 1986) y, por tanto, en la presente investigación se espera un resultado en la misma dirección (RE6).

Desde este tipo de abordajes, los hijos se entienden como un capital propio de la unión, en la medida en que los beneficios de tenerlos son mayores cuando se está con la pareja en comparación con no estar con ella (Becker; Landes; Michael, 1977). Sin embargo, el efecto de los hijos varía de acuerdo con el orden de los mismos. En Estados Unidos se encontró que el primero de ellos reduce el riesgo de divorcio, pero los siguientes lo incrementan (Lillard; Waite, 1993). Por el contrario, en Italia y España, el segundo o posterior hijo reduce el riesgo de ruptura (Coppola; Di Cesare, 2008). Adicionalmente, la edad de los hijos también influye sobre la separación, pues el riesgo es más bajo cuando estos se encuentran en edades preescolares (Lyngstad; Jalovaara, 2010; Bernardi; Martínez-Pastor, 2011).

La literatura latinoamericana confirma el efecto estabilizador de los hijos en la duración de la unión (Cabella, 2010; Miranda-Ribeiro, 1993; Pérez-Amador, 2008; Pérez-Amador; Ojeda, 2016; Santillán; Street, 2005), el cual, en el caso de México, se intensifica con cada hijo adicional (Solís; Ferraris, 2014). Respecto al número de hijos y edad de los mismos, proponemos que, en comparación con las parejas sin hijos, aquellas que si los tienen reportan un menor riesgo de ruptura. Esta asociación se observa con mayor fuerza cuando los hijos son pequeños (menos de 7 años) durante el tiempo de la unión (RE7). 


\section{Características del contexto de residencia}

En relación con la zona de residencia, dadas las fuertes presiones sociales que experimentan las parejas urbanas, en términos de la incertidumbre laboral y el empeoramiento de las condiciones de trabajo, así como el mayor contacto con ideas menos tradicionales de estos contextos en comparación con las zonas rurales y la menor presión social derivada del anonimato de las ciudades, se espera que la separación sea mayor en áreas urbanas (RE8), tal como desde los años setenta se ha venido confirmando en la región (Goldman, 1981; Ojeda, 1986; Pérez-Amador, 2008). Por otra parte, dada la difusión de la unión libre en todo el territorio nacional, no se esperan diferencias entre las regiones del país sobre el riesgo de ruptura de la primera unión (RE9).

\section{Metodología}

\section{Fuente de datos y análisis estadístico}

La información proviene de la Encuesta Nacional de Demografía y Salud (ENDS) de 2015, realizada por Profamilia y el Ministerio de Salud y Protección Social de Colombia (2017). El universo de estudio corresponde a la población civil, no institucionalizada, entre 13 y 69 años, de las áreas urbanas y rurales del país. La muestra es probabilística con representatividad de 6 regiones, 17 subregiones y 33 departamentos (incluyendo el Distrito Capital de Bogotá), en donde 52479 mujeres y 40300 hombres, entre 13 y 69 años, respondieron el cuestionario individual (Profamilia; Ministerio de Salud y Protección Social, 2017).

El presente estudio se basa en la historia de unión de los cuestionarios individuales de las mujeres en edades reproductivas, definidos por la encuesta como las personas entre 13 y 49 años (38 718 individuos). Sin embargo, dado que en el estudio de la ruptura de la unión solo las personas alguna vez unidas pueden experimentar el evento, se excluyeron 13272 casos de mujeres que nunca se habían unido en el momento de la encuesta. Considerando que la mayoría de los estudios revisados se basan en poblaciones de 15 o más años, también se excluyeron las personas menores de dicha edad (28 mujeres).

En una etapa posterior del procesamiento de los datos se excluyeron 151 registros de mujeres por no reportar los años de los eventos de interés. Cuando faltaba el mes del evento (unión o disolución), se asignó un mes aleatorio (de 1 a 12) con el propósito de no perder casos y contar con el mayor número de fechas de los eventos observados. Adicionalmente, se excluyeron los casos de uniones del mismo sexo ( 444 en la base de mujeres), con el fin de limitar el análisis únicamente a las uniones entre personas 
de sexo opuesto. Así, la muestra final en esta investigación está constituida por 24 823 mujeres en edades reproductivas.

La estrategia metodológica se realizó en tres etapas. La primera corresponde al cálculo de la distribución de las variables independientes en cada una de las poblaciones y subpoblaciones de interés. Para ello, los porcentajes se reportan teniendo en cuenta el diseño de la muestra y se indica el número de casos absolutos. La segunda y tercera etapas se desarrollaron mediante técnicas de supervivencia o de análisis de eventos (en inglés event history analysis). Estas técnicas permiten medir la relación entre determinadas características sociodemográficas de los individuos y el riesgo de disolución. El análisis tiene en cuenta los meses desde que cada individuo se une hasta que disuelve su primera unión. El tiempo de exposición con el que contribuyen las personas que no experimentaron el evento es considerado como episodios censurados a la derecha ${ }^{3}$, correspondiendo al tiempo transcurrido entre la primera unión y la fecha de la entrevista o la muerte de la pareja en los casos de viudez.

La primera parte de la segunda etapa corresponde al análisis de supervivencia a partir de estimaciones no paramétricas (Kaplan-Mier y función de la tasa instantánea de riesgo o hazard), disponibles en la suite de gráficos del módulo de Análisis de Supervivencia de Stata MP 12 (StataCorp, 2011). En ellas, se describen y comparan las pautas de ocurrencia del evento de interés sin tener en cuenta el efecto de las variables independientes. El cálculo de la supervivencia de la primera unión es la base para la estimación de la intensidad del fenómeno, es decir, para cuantificar el nivel de ruptura de la primera unión en el conjunto de la población colombiana. Es por esta razón que únicamente para este indicador, tanto nacional como departamental, se incluyen a los hombres de 15 a 59 años que habían experimentado la primera unión ( $\mathrm{n}=2 \mathrm{O}$ 927). Para el resto del análisis se tomó únicamente la muestra de mujeres descrita anteriormente.

Posteriormente, en la segunda parte de esta etapa, se realizan modelos multivariados que sí tienen en cuenta la relación de dichas variables con el riesgo de ruptura de la unión de las mujeres. Para el análisis multivariado se optó por el modelo exponencial constante a intervalos (ECI o piecewise constant exponencial model), ante la no proporcionalidad de los riesgos de disolución a lo largo del tiempo, requerida por el modelo tipo Cox ${ }^{4}$. Los modelos ECI son más flexibles que otras especificaciones

3. En las técnicas del análisis de supervivencia, los casos censurados a la derecha corresponden a la información incompleta del individuo, para quien se conoce la fecha de inicio del episodio, pero que al finalizar el período de observación aún no ocurría el evento de interés (Bernardi, 2006: 21).

4. Las pruebas de proporcionalidad de los riesgos (bajo una función exponencial y logarítmica) se realizaron para todas las variables independientes, siendo más de la mitad de ellas no significativas, entre ellas la cohorte y el tipo de unión. Por tales restricciones se usaron modelos ECI y no tipo Cox. 
(como los paramétricos Weibull o Gompertz), dado que no requieren ningún supuesto relacionado con la dependencia temporal del proceso (Bernardi; Martínez-Pastor, 2011). Por tanto, para nuestros modelos, la duración fue segmentada en 10 períodos de 24 meses y uno adicional con las duraciones posteriores a 240 meses o 20 años. Bajo los modelos ECI, se asume una tasa de transición constante en cada segmento, pero que cambia entre ellos.

En la segunda parte de la segunda etapa del análisis, se probaron 4 modelos para seleccionar aquel que ofreciera la mejor solución en términos de la bondad de ajuste (a partir del valor del AIC ${ }^{5}$ y del Log-Likelihood) y de la claridad conceptual para los fines de los objetivos aquí propuestos (Escobar; Fernández; Bernardi, 2009). En el primero de ellos (modelo 1) solo se incluye la variable de la cohorte de la unión a fin de comparar el riesgo de separación de las uniones recientes. Posteriormente, se agrega el tipo de unión (modelo 2) para observar si hay un cambio en el riesgo de las cohortes cuando se controla por el arreglo conyugal (matrimonio vs. unión libre). En el modelo 3 se adicionan todas las variables sociodemográficas y se constata nuevamente el riesgo de las cohortes. Para finalizar esta fase, se incluye una interacción entre la cohorte y el tipo de unión con el objetivo de identificar si la asociación de la cohorte con la separación está mediada por un efecto del tipo de unión (modelo 4).

\section{Variables independientes}

Las variaciones en los niveles y riesgos asociados a la ruptura de la unión se analizan a través de las variables independientes que se describen a continuación.

- Cohorte de unión: es una variable con tres categorías de acuerdo con dos momentos importantes en la regulación de las uniones conyugales en Colombia, a saber:

- Antes de 1991: corresponde a las uniones conformadas entre 1977 y 1990, cuando aún persistían diferencias jurídicas considerables entre el matrimonio y la unión libre. Es la categoría de referencia en los modelos multivariados.

- 1991-2003: uniones acontecidas desde 1991 cuando se aprobó la Ley de la Unión Marital de Hecho y se homologó la unión libre al matrimonio en gran parte de sus derechos y obligaciones. Dado que la Ley fue promulgada el 28 de diciembre de 1990, esta categoría incluye las uniones a partir de 1991, cuando ya es vigente la

5. El AIC (Akaike Information Criteria) es una medida de la calidad relativa de un modelo usado para comparar la bondad de ajuste de distintos modelos. Se calcula utilizando la verosimilitud y el número de parámetros del modelo, y su valor es interpretable en la comparación entre distintos modelos. El mejor de ellos es aquel con el AIC más bajo (Escobar; Fernández; Bernardi, 2009). Se ha empleado en estudios sobre el divorcio, como por ejemplo Bernardi y Martínez-Pastor (2011). 
ley. El 2003 se estableció por ser un año intermedio entre 1990 y 2016, año de las uniones más recientes.

- 2004-2016: corresponde a las uniones conformadas entre 2004 y $2016^{6}$.

- Tipo de unión: matrimonio y unión libre. En los modelos esta información se incluye como variable que cambia en el tiempo, lo cual permite que el tiempo de las uniones libres que luego transitan a un matrimonio sea dividido en dos episodios. El primero abarca el período entre el inicio de la unión y la fecha del matrimonio con la misma pareja (definidos como casos censurados a la derecha). El segundo cubre el período entre el matrimonio y la fecha de separación o de censura cuando no experimenta el evento. Si bien este procedimiento incrementa los casos bajo observación, al declarar la base como un objeto biográfico, el programa estadístico tiene en cuenta la variable de identificación individual, evitando con ello la duplicación del tiempo de exposición total de una misma persona. En los modelos multivariados, el matrimonio se toma como categoría de referencia.

- Nivel educativo: fue elaborada a partir de los grados de escolaridad, con cuatro categorías: 1) estudios primarios completos o menos (categoría de referencia); 2) secundaria incompleta; 3) secundaria completa y 4) estudios superiores.

- Edad a la unión: edad de la entrevistada al momento de la primera unión.

- Homogamia de edad: corresponde a los años de diferencia de edad con la pareja de la primera unión. A partir de allí se construyó una variable con cinco categorías: 1) edad similar, es decir, que existen menos de dos años de diferencia entre los miembros de la pareja; 2) mujer mayor que el hombre 3 o más años; 3 ) mujer menor que el hombre de 3 a 9 años; 4) mujer menor que el hombre 10 o más años, y 5) sin información, en los casos donde la persona no sabía la edad de la pareja. El criterio de 2 años se adopta como un recurso analítico que a su vez permite ver las variaciones a lo largo de las cohortes de unión. La categoría homogamia de edad es la categoría de referencia en los modelos multivariados.

- Paridad y edad del hijo menor de la mujer: es una variable categórica que cambia en el tiempo no solo en términos del número de hijos, sino de la edad del menor de ellos cuando son dos o más. Esta variable se construyó siguiendo ejercicios similares en Italia (Salvini; Vignoli, 2O11; Vignoli; Ferro, 2009) y España (Bernardi; Martínez-Pastor, 2O11), en donde se tiene en cuenta el cambio únicamente hasta el segundo o posterior hijo. Sin embargo, dado el mayor nivel de fecundidad de Colombia, especialmente en las cohortes más antiguas, se creó una variable que tiene en cuenta hasta el cuarto hijo (mujeres que al menos tuvieron un cuarto hijo). De esta manera, se incluyen 9 categorías:

6. Aunque la ENDS tiene el 2015 como año de referencia, su trabajo de campo se extendió hasta 2016. Por ello, las historias de unión pueden incluir fechas en dicho año. 
- Ningún hijo (categoría de referencia en los modelos multivariados).

- Un hijo menor de 7 años en cada mes de duración de la unión hasta la ruptura, o hasta la fech a de la encuesta o la muerte de la pareja. Las dos últimas situaciones se tratan como valores censurados a la derecha .

- Un hijo con 7 o más años...

- Dos hijos, y el menor tiene menos de 7 años...

- Dos hijos, y el menor tiene 7 o más años...

- Tres hijos, y el menor tiene menos de 7 años...

- Tres hijos, y el menor tiene 7 o más años...

- Al menos cuatro hijos, y el menor tiene menos de 7 años...

- Al menos cuatro hijos, y el menor tiene 7 o más años...

Hay que tener en cuenta que esta es una variable individual de la mujer, es decir, no es posible establecer si los hijos son o no de la pareja, siendo posible que las mujeres iniciaran la primera unión con hijos. Sin embargo, dado que el interés se centra en conocer el efecto de tener un determinado número de hijos y la edad de estos sobre el riesgo de la separación, esta variable se concibe como un atributo individual de la mujer y no de la unión. La encuesta tampoco permite identificar si los hijos estuvieron presentes o no en la dinámica de la pareja, pero en la mayoría de los casos se asume que sí.

- Fecundidad previa a la unión: variable dicotómica que asume el valor de 1 cuando al menos un hijo nació antes de la fecha de la unión y o cuando no es así.

- Zona de residencia actual: se incluye como una variable proxy, dado que no se cuenta con esta información al momento de la unión o la disolución. Tiene dos valores: vivir actualmente en una zona urbana o en una rural (definida como categoría de referencia en los modelos multivariados).

- Región de residencia actual: similar a la zona de residencia, esta variable es un proxy de la ubicación geográfica actual del individuo, en 6 regiones: Atlántica, Bogotá, Central, Oriental, Orinoquía/Amazonía y Pacífica. La región Oriental es la categoría de referencia en los modelos multivariados.

- Otras variables de caracterización actual: además de las anteriores variables, se consideraron la edad actual, el estado civil actual y el número de uniones como variables que describen la población bajo estudio en términos de sus características actuales, pero no incluidas en los modelos por no responder a los objetivos aquí planteados.

7. Las cursivas indican que se repite lo mismo en las siguientes categorías, a partir de los puntos suspensivos. 


\section{Resultados}

Los hallazgos del estudio se presentan en tres apartados. En el primero se describen las principales características sociodemográficas de la muestra de mujeres entre 15 y 49 años que se unieron al menos una vez, para quienes se observa la disolución de su primera unión. También se describe cómo tales características han cambiado a lo largo de las cohortes de unión aquí comparadas, dando elementos para comprender los resultados con relación a la supervivencia de las uniones y los riesgos asociados a su disolución, presentados en la segunda y tercera sección, respectivamente.

\section{Cambios generacionales en los patrones de nupcialidad desde el estudio de la disolución de las uniones}

Antes de describir cómo las cohortes han cambiado en términos de los patrones de formación de la primera unión y de la fecundidad, se presenta una descripción general de la población, en términos de las características que las personas alguna vez unidas reportan al momento de la encuesta. De acuerdo con la Tabla 1, un poco más del $51 \%$ se encuentran en los grupos de edad 25-29, 30-34 y 35-39 años. Resalta que casi dos terceras partes de las mujeres cuentan con algún estudio superior (32 $\%)$, siendo el nivel más frecuente de escolaridad. En términos de la zona y la región de residencia, se observa que la mayoría vivían en áreas urbanas ( $78 \%$ y alrededor del $46 \%$ vivía en las regiones Central y Atlántica.

$\mathrm{Al}$ estudiar la primera ruptura de la unión se incluye la experiencia de todas las mujeres que al menos se unieron una vez. Sin embargo, la variable sobre el número de uniones, indica que $24 \%$ de las mujeres se vuelven a unir, lo cual hace que, en la actualidad, un porcentaje importante $(77 \%)$ declare un estado civil de unión, siendo la unión libre el más frecuente (50 \% vs. $27 \%$ de los matrimonios). Adicionalmente, una quinta parte de la muestra declara estar separada o divorciada en este momento (21\%), siendo mínima la viudez por ser un grupo de mujeres en edades jóvenes.

Cuando tomamos como punto de referencia la experiencia de la primera unión, también se observan diferencias interesantes entre las cohortes de unión. La distribución de las variables independientes para cada cohorte de unión se incluye en la Tabla 2. Una primera diferencia radica en la distribución de las cohortes observadas. Dado que la muestra incluye únicamente mujeres hasta los 49 años, el porcentaje de uniones formadas antes de 1991 es muy bajo (14\%) en comparación con las cohortes más recientes. Cuando se analiza la distribución del estado actual de la primera unión a lo largo de las tres cohortes, se evidencian las ganancias en la esperanza de vida al observar que la viudez va perdiendo participación en el tiempo, pues mientras 
\begin{tabular}{l|l} 
TABLA 1 & $\begin{array}{l}\text { Distribución de las variables al momento de la encuesta. } \\
\text { Mujeres 15-49 (Colombia, 2015) }\end{array}$
\end{tabular}

\section{Variable}

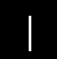

Casos

Porcentaje

$\mathbf{n}=\mathbf{2 4 8 2 3}$

\section{Edad}

\begin{tabular}{|c|c|c|}
\hline $15-19$ & 1227 & 4,0 \\
\hline $20-24$ & 3258 & 12,8 \\
\hline $25-29$ & 4092 & 17,3 \\
\hline $30-34$ & 4350 & 17,5 \\
\hline $35-39$ & 4129 & 16,7 \\
\hline $40-44$ & 3883 & 15,8 \\
\hline $45-49$ & 3884 & 15,9 \\
\hline
\end{tabular}

\section{Nivel}

\begin{tabular}{lcc} 
Primaria o menos & 6752 & 22,5 \\
\hline Secundaria incompleta & 5068 & 19,6 \\
\hline Secundaria completa & 6239 & 26,2 \\
\hline Superior & 6764 & 31,7
\end{tabular}

\section{Estado civil}

\begin{tabular}{lcc} 
En unión libre & 13479 & 50,6 \\
\hline Casada & 5815 & 26,6 \\
\hline Separada & 4837 & 19,8 \\
\hline Divorciada & 196 & 1,0 \\
\hline Viuda & 496 & 2,0
\end{tabular}

\section{Número de uniones}

\begin{tabular}{|c|c|c|}
\hline 1 & 18236 & 75,6 \\
\hline 2 & 5311 & 20,2 \\
\hline $3^{+}$ & 1276 & 4,2 \\
\hline \multicolumn{3}{|c|}{ Zona de residencia } \\
\hline Rural & 6631 & 22,0 \\
\hline Urbana & 18192 & $78, \mathrm{o}$ \\
\hline
\end{tabular}




\begin{tabular}{|lcc}
\hline \multicolumn{2}{l}{ Región de residencia } & \multicolumn{1}{c}{} \\
\hline Oriental & 3416 & 17,1 \\
\hline Atlántica & 6457 & 22,3 \\
\hline Central & 5510 & 24,0 \\
\hline Pacífica & 3894 & 17,3 \\
\hline Bogotá & 1449 & 16,8 \\
\hline Territorios Nacionales & 4097 & 2,5 \\
\hline
\end{tabular}

Nota: Los casos corresponden al número original de la encuesta. Los porcentajes se reportan con valores ponderados.

Fuente: cálculos propios a partir de la ENDS 2015 (Profamilia; Ministerio de Salud y Protección Social, 2017).

en la cohorte más antigua fue del $8 \%$, en la cohorte más joven fue del $1 \%$. Si bien el tiempo de exposición no es el mismo para ambas cohortes, la cohorte intermedia (1991-2003) también declaraba valores menores, confirmando así esta tendencia.

En segundo lugar, se observa el protagonismo de la unión libre como el arreglo conyugal más frecuente en las cohortes más jóvenes, a expensas de la unión libre prematrimonial (irse a vivir con la pareja antes de casarse) y el matrimonio directo. Mientras de las uniones iniciadas entre 2004 y 2016, el 14 \% iniciaron bajo un matrimonio, en la cohorte más antigua este porcentaje llegaba casi al $26 \%$. De igual manera, el predominio de la unión consensual también se confirma desde un enfoque biográfico, teniendo en cuenta los meses que toda la muestra experimentó en la primera unión (alrededor de 3000 ooo, tal como consta en la parte final de la Tabla 2). De este tiempo, de la cohorte de unión anterior a 1991 transcurrió el $41 \%$ en matrimonio, en comparación con las uniones más recientes que lo han hecho en $19 \%$.

Además de los cambios en las preferencias por los tipos de unión, se han dado transformaciones importantes relacionadas con los calendarios de la formación de la unión. La edad de la primera unión ha venido aumentando progresivamente, lo cual se confirma en la menor participación del grupo de mujeres unidas antes de los 18 años, que en la primera cohorte llegaba casi al $50 \%$ y en la última fue del $27 \%$. En el caso de las mujeres que formaron su primera unión con 25 o más años, se observa un aumento sin precedentes, al pasar de menos del $1 \%$ al $22 \%$ entre las cohortes extremas. Por otra parte, en este contexto de la postergación de los calendarios de la formación de parejas, también se confirma una disminución de la diferencia de edad con la pareja, cuando el hombre es mayor que ella (este grupo alcanzó el 72 $\%$ en la cohorte más antigua, reduciéndose a $58 \%$ en las uniones más jóvenes). Sin embargo, el grupo de mujer mayor que el hombre, aumentó de manera importante, pues de contribuir $1 \%$ ahora lo hace en $6 \%$ en las uniones más jóvenes. 
Distribución de las variables independientes fijas y cambiantes en

TABLA 2 el tiempo, relacionadas con la primera unión. Mujeres 15-49 años (Colombia, 2015)

\section{Variables \\ Todas las \\ uniones $(n=24823)$}

\section{Cohorte de la unión}

$\begin{array}{ccc}\text { Antes de 1991 } & 1991-2003 & 2004-2016 \\ (n=3873) & (n=10292) & (n=10658)\end{array}$

VARIABLES FIJAS EN EL TIEMPO

\section{Cohorte de la unión}

\begin{tabular}{lc} 
Antes de 1991 & 14,3 \\
\hline $1901-2003$ & 41,5 \\
\hline $2004-2016$ & 44,3
\end{tabular}

\section{Estado actual de la primera unión}

\begin{tabular}{lcccc}
\hline En unión & 58,8 & 44,3 & 52 & 69,9 \\
\hline $\begin{array}{l}\text { Disuelta por separación } \\
\text { o divorcio }\end{array}$ & 38 & 47,8 & 44,1 & 29 \\
\hline Disuelta por viudez & 3,2 & 8 & 3,9 & 1,1 \\
\hline
\end{tabular}

Tipo de arreglo conyugal al inicio de la primera unión

\begin{tabular}{lcccc}
\hline Matrimonio directo & 19,3 & 25,4 & 23,1 & 13,7 \\
\hline Unión libre prematrimonial & 12,6 & 18,1 & 15,1 & 8,4 \\
\hline Unión libre permanente & 68,2 & 56,5 & 61,9 & 77,9 \\
\hline
\end{tabular}

Edad al momento de la primera unión

\begin{tabular}{lcccc} 
Menos de 18 años & 32,3 & 49,2 & 31,9 & 27,2 \\
\hline $18-24$ años & 50,9 & 50,7 & 51,5 & 50,5 \\
\hline 25 o más años & 16,8 & 0,1 & 16,6 & 22,3
\end{tabular}

Homogamia de edad en la primera unión

\begin{tabular}{lcccc} 
Edad similar (2 años o menos) & 32,4 & 24 & 32,6 & 35 \\
\hline $\begin{array}{l}\text { Mujer mayor que el hombre } \\
\text { 3 o más años }\end{array}$ & 5,2 & 1,3 & 5,3 & 6,4 \\
\hline $\begin{array}{l}\text { Mujer menor que el hombre } \\
\text { 3-9 años }\end{array}$ & 42,1 & 49,1 & 41,2 & 40,7 \\
\hline $\begin{array}{l}\text { Mujer menor que el hombre } \\
\text { 10 o más años }\end{array}$ & 19,1 & 23,4 & 19,7 & 17,2 \\
\hline Sin información & 1,2 & 2,1 & 1,3 & 0,7 \\
\hline
\end{tabular}




\begin{tabular}{|c|c|c|c|c|}
\hline \multicolumn{5}{|c|}{ Fecundidad previa a la primera unión } \\
\hline No & 78,5 & 87,3 & 80 & 74,4 \\
\hline Sí, al menos un hijo & 21,5 & 12,7 & 20 & 25,6 \\
\hline \multicolumn{5}{|c|}{ VARIABLES QUE CAMBIAN EN EL TIEMPO } \\
\hline \multicolumn{5}{|c|}{ Tipo de arreglo durante la primera unión } \\
\hline Matrimonio & 33,5 & 41,5 & 34,4 & 19,1 \\
\hline Unión libre & 66,5 & 58,5 & 65,6 & 80,9 \\
\hline \multicolumn{5}{|c|}{ Paridad y edad del hijo menor durante la primera unión } \\
\hline Ninguno & 14,7 & 9,3 & 12,9 & 27,6 \\
\hline 1 hijo $<7$ años & 22,3 & 13,7 & 20,9 & 38,8 \\
\hline 1 hijo $\geq 7$ años & 6,6 & 4,3 & 7,8 & 6,8 \\
\hline 2 hijos $<7$ años & 19,9 & 17 & 22,2 & 18,2 \\
\hline 2 hijos $\geq 7$ años & 7,7 & 9 & 9,2 & 1,8 \\
\hline 3 hijos $<7$ años & 10,9 & 14,1 & 11,5 & 4,6 \\
\hline 3 hijos $\geq 7$ años & 4,9 & 7,5 & 5 & 0,6 \\
\hline 4 hijos o más $<7$ años & 6,5 & 10,8 & 6 & 1,2 \\
\hline 4 hijos o más $\geq 7$ años & 6,6 & 14,4 & 4,5 & $\mathrm{O}, 4$ \\
\hline Meses de exposición & 3097397 & 898844 & 1594567 & 603986 \\
\hline Mujeres & 24823 & 3873 & 10292 & 10658 \\
\hline Rupturas & 9608 & 1847 & 4616 & 3145 \\
\hline
\end{tabular}

Fuente: cálculos propios a partir de la ENDS 2015 (Profamilia; Ministerio de Salud y Protección Social, 2017).

Finalmente, las descripciones de las cohortes en términos de sus patrones de fecundidad también evidencian las transformaciones familiares que ha experimentado el país. Uno de estos cambios es la apertura progresiva de la fecundidad previa a la primera experiencia de unión. Tener al menos un hijo antes de la primera unión fue la situación de $13 \%$ de las mujeres de las cohortes previas a 1991, y del 26 $\%$ de las uniones formadas después de 2003. Aunque es posible que un porcentaje importante de estas mujeres formaran parejas con los padres de sus hijos, el hecho de que los hijos nazcan antes de establecerse la unión expresa un cambio cualitativo en la relación entre fecundidad y conyugalidad. 
Por otra parte, aunque ahora son más frecuentes los hijos previos a la unión, las mujeres pasan menos tiempo con tres o cuatro hijos, o incluso sin ellos. Teniendo en cuenta todos los meses transcurridos entre la unión y la disolución o el momento de la encuesta, en la cohorte de unión previa a 1991 las mujeres pasaron tan solo el $9 \%$ del tiempo sin hijos; en la cohorte 2004-2016 este valor fue de $28 \%$. Un incremento similar se observó en los meses con un hijo menor de 7 años (de 14 \% a $39 \%$ ), o con dos hijos y el menor de ellos también con menos de 7 años (de $17 \%$ a $18 \%$ ). A partir del tercer hijo las reducciones son considerables, haciendo que, entre las dos cohortes comparadas, las mujeres con tres y cuatro o más hijos, redujeran su tiempo en esta situación 40 puntos porcentuales (de $47 \%$ al $7 \%$ ), aunque deben tenerse en cuenta las diferencias en el tiempo de exposición de cada cohorte.

\section{Nuevas estimaciones de la ruptura de la primera unión y sus variaciones departamentales}

A continuación, se presentan las estimaciones nacionales y departamentales de la supervivencia de las uniones ${ }^{8}$. La no supervivencia corresponde a la disolución de la primera unión, la cual fue estimada para cada año de duración a nivel nacional, y para duraciones específicas (6o, 120, 180 y 240 meses, equivalentes a los años 5, 10, 15 y 20 de unión). De acuerdo con la Tabla 3, el $6 \%$ de las uniones se habían disuelto tras el primer año de haber iniciado. En los años 5, 10, 15 y 20, estos niveles fueron del 23, 35, 41 y $48 \%$, respectivamente. Cuando se analiza la supervivencia por tipo de unión, se observa que la disolución de los matrimonios es mucho menor al de la unión libre a lo largo de todas las duraciones. Por ejemplo, al quinto año de unión (mes 6o) ya se habían disuelto $26 \%$ de estas últimas frente al $7 \%$ de los matrimonios; es decir, un valor 3,8 veces mayor. En los primeros años, las brechas son grandes entre los dos arreglos conyugales, pero se van acortando progresivamente, hasta que en la duración 20 años (mes 240), el nivel de disolución de la unión libre es 2,1 veces mayor que el del matrimonio.

Además de las diferencias por tipo de unión también existen variaciones territoriales importantes. Dado el limitado tamaño de las muestras, los trabajos disponibles para Colombia (Goldman, 1981; Zamudio; Rubiano, 1991) no ofrecían estimaciones del nivel departamental, haciendo que nuestras estimaciones fueran las primeras en este nivel territorial. La Tabla 4 describe la supervivencia de las uniones en los años 5, 10, 15 y 20 para todos los departamentos del país. En todas las duraciones,

8. Con el fin de obtener estimaciones del conjunto de la población, la supervivencia de las uniones incluye también a los hombres entre 15 y 59 años. La información de estos proviene del cuestionario individual de hombres de la ENDS 2015 (Profamilia; Ministerio de Salud y Protección Social, 2017). 
\begin{tabular}{|l|l|}
\hline TABLA 3 & $\begin{array}{l}\text { Supervivencia de las uniones (proporción). Mujeres 15-49 años } \\
\text { y hombres 15-59 años (Colombia, 2015), (n=45 750) }\end{array}$ \\
\hline
\end{tabular}

\begin{tabular}{|c|c|c|c|c|c|}
\hline $\begin{array}{l}\text { Duración } \\
\text { (meses) }\end{array}$ & $\begin{array}{l}\text { Duración } \\
\text { (años) }\end{array}$ & $\begin{array}{l}\text { Todas las } \\
\text { uniones }\end{array}$ & $\begin{array}{l}\text { Matrimonio } \\
\text { (M) }\end{array}$ & $\begin{array}{c}\text { Unión libre } \\
\text { (UL) }\end{array}$ & $\begin{array}{c}\text { Diferencia } \\
\text { M-UL }\end{array}$ \\
\hline 12 & 1 & 0,94 & 0,99 & 0,93 & 0,06 \\
\hline 24 & 2 & 0,89 & 0,97 & 0,87 & 0,10 \\
\hline 36 & 3 & 0,84 & 0,96 & 0,82 & 0,14 \\
\hline 48 & 4 & 0,80 & 0,94 & 0,77 & 0,17 \\
\hline 60 & 5 & 0,77 & 0,93 & 0,74 & 0,20 \\
\hline 72 & 6 & 0,74 & 0,91 & 0,70 & 0,21 \\
\hline 84 & 7 & 0,71 & 0,90 & 0,67 & 0,23 \\
\hline 96 & 8 & 0,69 & 0,89 & 0,65 & 0,24 \\
\hline 108 & 9 & 0,67 & 0,88 & 0,63 & 0,25 \\
\hline 120 & 10 & 0,65 & 0,86 & 0,60 & 0,26 \\
\hline 132 & 11 & 0,63 & 0,85 & 0,58 & 0,27 \\
\hline 144 & 12 & 0,62 & 0,84 & 0,57 & 0,27 \\
\hline 156 & 13 & 0,60 & 0,83 & 0,55 & 0,28 \\
\hline 168 & 14 & 0,59 & 0,81 & 0,54 & 0,28 \\
\hline 180 & 15 & 0,57 & 0,80 & 0,52 & 0,28 \\
\hline 192 & 16 & 0,56 & 0,79 & 0,51 & 0,28 \\
\hline 204 & 17 & 0,55 & 0,78 & 0,50 & 0,28 \\
\hline 216 & 18 & 0,54 & 0,77 & 0,49 & 0,28 \\
\hline 228 & 19 & 0,53 & 0,76 & 0,48 & 0,28 \\
\hline 240 & 20 & 0,52 & 0,75 & 0,47 & 0,28 \\
\hline
\end{tabular}

Fuente: elaboración propia a partir de la ENDS 2015 (Profamilia; Ministerio de Salud y Protección Social, 2017).

Boyacá y Nariño son los lugares con menores niveles de disolución, seguidos de Huila y Santander, que se ubican en el tercer o cuarto lugar de mayor supervivencia. En el extremo opuesto se observan Arauca, Chocó y Amazonas como los departamentos de mayor ruptura de la unión en las tres primeras duraciones observadas. En el largo plazo (20 años), el valor de disolución más alto lo registran Arauca (60 \%), Vichada $(58 \%)$ y Meta $(57 \%)$. 
Las estimaciones también permiten apreciar la desigual distribución de la disolución de las uniones en los departamentos. Por ejemplo, en la duración 5 años Boyacá registró un nivel de disolución de $14 \%$, mientras en Chocó fue del $33 \%$. Algo similar ocurre en las duraciones avanzadas (20 años) con Boyacá y Arauca, que reportaron valores de 31 y $60 \%$, respectivamente. Estas diferencias en los departamentos pueden originarse por distintas razones, considerando que los patrones de nupcialidad también son diversos en el territorio. La intensidad de la unión libre no es igual en estos lugares, así como tampoco el nivel de fecundidad o la participación laboral de las mujeres, lo cual puede afectar el riesgo individual de experimentar una ruptura. Es por esto que, en la siguiente sección, se presentan los resultados de los modelos multivariados que explican el riesgo de disolución cuando se controla simultáneamente por el efecto de variables sociodemográficas, entre ellas, la región de residencia.

TABLA 4 Supervivencia departamental de las uniones (proporción). Mujeres 15-49 años y hombres 15-59 años (Colombia, 2015), ( $\mathrm{n}=45750)$

\begin{tabular}{l|cccc}
\hline Departamento (años) & $\mathbf{1 5}$ & $\mathbf{2 0}$ \\
\hline Amazonas & $\mathbf{5}$ & $\mathbf{1 0}$ & $\mathbf{1 5}$ & 0,46 \\
\hline Antioquia & 0,70 & 0,56 & 0,50 & 0,52 \\
\hline Arauca & 0,77 & 0,66 & 0,58 & 0,40 \\
\hline Atlántico & 0,70 & 0,54 & 0,43 & 0,55 \\
\hline Bogotá & 0,78 & 0,67 & 0,60 & 0,51 \\
\hline Bolívar & 0,77 & 0,65 & 0,56 & 0,47 \\
\hline Boyacá & 0,73 & 0,60 & 0,53 & 0,69 \\
\hline Caldas & 0,86 & 0,78 & 0,73 & 0,56 \\
\hline Caquetá & 0,80 & 0,68 & 0,62 & 0,52 \\
\hline Casanare & 0,78 & 0,64 & 0,57 & 0,56 \\
\hline Cauca & 0,79 & 0,67 & 0,60 & 0,57 \\
\hline Cesar & 0,79 & 0,68 & 0,61 & 0,50 \\
\hline Chocó & 0,74 & 0,63 & 0,56 & 0,43 \\
\hline Córdoba & 0,67 & 0,55 & 0,48 & 0,60 \\
\hline Cundinamarca & 0,78 & 0,68 & 0,63 & 0,56 \\
\hline Guainía & 0,81 & 0,71 & 0,63 & 0,60 \\
\hline
\end{tabular}




\begin{tabular}{lcccc} 
Guaviare & 0,75 & 0,59 & 0,51 & 0,46 \\
\hline Huila & 0,82 & 0,74 & 0,68 & 0,63 \\
\hline La Guajira & 0,73 & 0,61 & 0,51 & 0,48 \\
\hline Magdalena & 0,77 & 0,66 & 0,60 & 0,56 \\
\hline Meta & 0,73 & 0,58 & 0,49 & 0,42 \\
\hline Nariño & 0,83 & 0,75 & 0,69 & 0,65 \\
\hline Norte de Santander & 0,77 & 0,64 & 0,58 & 0,52 \\
\hline Putumayo & 0,80 & 0,68 & 0,61 & 0,57 \\
\hline Quindío & 0,73 & 0,60 & 0,48 & 0,44 \\
\hline Risaralda & 0,78 & 0,63 & 0,55 & 0,48 \\
\hline San Andrés y Providencia & 0,72 & 0,58 & 0,50 & 0,44 \\
\hline Santander & 0,83 & 0,73 & 0,66 & 0,62 \\
\hline Sucre & 0,77 & 0,67 & 0,60 & 0,55 \\
\hline Tolima & 0,82 & 0,69 & 0,62 & 0,55 \\
\hline Valle & 0,74 & 0,60 & 0,51 & 0,45 \\
\hline Vaupés & 0,80 & 0,70 & 0,65 & 0,60 \\
\hline Vichada & 0,76 & 0,60 & 0,52 & 0,42 \\
\hline & & 0 & 0 \\
\hline
\end{tabular}

Fuente: elaboración propia a partir de la ENDS 2015 (Profamilia; Ministerio de Salud y Protección Social, 2017).

\section{Los diferenciales sociodemográficos de la ruptura de la unión}

Dado que las curvas de supervivencia no tienen en cuenta la asociación de otras variables relacionadas con la fecundidad, nupcialidad, escolaridad y territorio, se presentan los modelos multivariados para explorar la relación de la cohorte y el tipo de unión sobre el riesgo de separación conyugal. Los hallazgos se presentan a partir de los modelos incluidos en la Tabla 5. El modelo que incluye únicamente la cohorte de la unión (modelo 1) identifica los mismos resultados de las curvas de supervivencia de acuerdo con esta variable, es decir, un mayor riesgo de disolución de las uniones más jóvenes en comparación con las iniciadas antes de 1991. Al incluir el tipo de unión (modelo 2), los riesgos de las cohortes disminuyen levemente, pero siguen confirmando la mayor probabilidad de ruptura de las cohortes más jóvenes $\mathrm{y}$, al mismo tiempo, de las uniones libres en comparación con los matrimonios. Adicionalmente, se observa una mejora del valor AIC, indicando que, en efecto, el tipo de unión es una variable relevante. 
Cuando se incluyen las variables sociodemográficas (modelo 3), el valor AIC vuelve a disminuir (de 54606,5 a 53 806,4), confirmando la contribución de dichas variables para explicar la disolución de las uniones. Bajo esta versión completa del modelo, las razones de riesgo de las cohortes vuelven a aumentar, y las del tipo de unión disminuyen un poco. De esta manera, a partir de los resultados del modelo tres, se encontró que, en comparación con la cohorte anterior a 1991, las uniones formadas entre 1991 y 2003 tienen un riesgo de disolución $23 \%$ más grande, y en la cohorte 2004-2016 este riesgo es incluso más elevado (73\%).

Asimismo, se confirma el mayor riesgo de disolución de las uniones libres, el cual es 2,1 veces más elevado que el de los matrimonios. Sin embargo, este mayor riesgo de ruptura de las uniones libres solo es estadísticamente significativo para la cohorte 2004-2016, a juzgar por los resultados de la interacción entre cohorte y tipo de unión (modelo 4), los cuales aportan evidencia estadística para afirmar que las uniones libres más recientes tienen mayor riesgo de disolución que las uniones libres anteriores a 1991, cuando se las compara con los matrimonios de ambas cohortes. Al comparar el valor AIC, se observa una leve disminución (de 53 806,4 a 53 801,4), indicando que la cohorte tiene un papel importante en la relación entre el tipo de unión y la separación.

En términos de los otros diferenciales (modelos 3 y 4 de la Tabla 5), es posible afirmar que cada año adicional de postergación de la unión reduce de forma significativa el riesgo de disolución. Adicionalmente, otras características que disminuyen el riesgo de ruptura son las uniones entre una mujer y un hombre en donde él es mayor 3-9 años, y tener 203 hijos menores de 7 años, o 4 hijos sin importar su edad; en comparación con los individuos que se unen con parejas de edades similares y no tienen hijos. Por su parte, las características que aumentan el riesgo de ruptura corresponden a las uniones en donde la mujer es 3 o más años mayor, haber tenidos hijos antes de la unión, tener estudios secundarios o superiores, vivir en zonas urbanas y en las regiones Atlántica, Central y Pacífica; respecto a las personas que se unen con alguien de edad similar, no tiene hijos en la primera unión ni antes de ella, tiene estudios primarios o menos, viven en zonas rurales y en la región Oriental. 
\begin{tabular}{|l|l|l|l|} 
Modelo 1 & Modelo 2 & Modelo 3 & Modelo 4
\end{tabular}

Cohorte de la unión (ref: Antes de 1991)

\begin{tabular}{ccccc}
$1990-2003$ & $1,19^{* *}$ & $1,17^{* *}$ & $1,23^{* *}$ & $1,23^{* * *}$ \\
\hline $2004-2016$ & $1,76^{* * *}$ & $1,63^{* *}$ & $1,73^{* * *}$ & $1,42^{* * *}$
\end{tabular}

\section{Tipo de unión (ref: matrimonio)}

\begin{tabular}{|c|c|c|}
\hline Unión libre & $2,07^{* * *}$ & $1,99^{* * *}$ \\
\hline Edad a la unión & $0,94^{* * *}$ & $0,94^{* * *}$ \\
\hline \multicolumn{3}{|l|}{ Homogamia de edad (ref: edad similar ( $<3$ años) } \\
\hline Mujer mayor que el hombre 3 o más años & $1,10^{*}$ & $1,10^{*}$ \\
\hline Mujer menor que el hombre 3-9 años & $0,90^{* * *}$ & $0,90^{* * *}$ \\
\hline Mujer menor que el hombre 10 o más años & 0,99 & 0,99 \\
\hline Sin información & $1,27^{* * *}$ & $1,28^{* * *}$ \\
\hline \multicolumn{3}{|c|}{ Paridad y edad del hijo menor de la mujer (ref: ninguno) } \\
\hline 1 hijo $<7$ años & $1, \mathrm{O} 3$ & 1,02 \\
\hline 1 hijo $\geq 7$ años & 1,07 & 1,06 \\
\hline 2 hijos $<7$ años & $0,88^{* * *}$ & $0,88^{* * *}$ \\
\hline 2 hijos $\geq 7$ años & $1, \mathrm{O} 2$ & $1, \mathrm{O} 2$ \\
\hline 3 hijos $<7$ años & $0,79^{* * *}$ & $0,79^{* * *}$ \\
\hline 3 hijos $\geq 7$ años & 0,94 & 0,94 \\
\hline 4 hijos o más $<7$ años & $0,73^{* * *}$ & $0,73^{* * *}$ \\
\hline 4 hijos o más $\geq 7$ años & $0,74^{* * *}$ & $0,75^{* * *}$ \\
\hline \multicolumn{3}{|l|}{ Fecundidad previa a la unión (ref: no) } \\
\hline Sí & $1,23^{* * *}$ & $1,23^{* * *}$ \\
\hline \multicolumn{3}{|l|}{ Nivel educativo (ref: primaria o menos) } \\
\hline Secundaria incompleta & $1,12^{* * *}$ & $1,12^{* * *}$ \\
\hline Secundaria completa & $1, \mathrm{O} 8^{* *}$ & $1,08^{* *}$ \\
\hline Superior & $1,25^{* * *}$ & $1,25^{* * *}$ \\
\hline
\end{tabular}


Zona de residencia actual (ref: rural)

Urbana

Región de residencia actual (ref: Oriental)

\begin{tabular}{|c|c|c|}
\hline Atlántica & $1,10^{* *}$ & $1,10^{* * *}$ \\
\hline Central & $1,13^{* * *}$ & $1,13^{* * *}$ \\
\hline Pacífica & $1,13^{* * *}$ & $1,13^{* * *}$ \\
\hline Bogotá & 1,06 & 1,06 \\
\hline Orinoquía y Amazonía & $1, \mathrm{O} 1$ & $1, \mathrm{O} 2$ \\
\hline
\end{tabular}

Cohorte* Tipo unión (ref: cohorte previa 1991 y matrimonios)

Cohorte 1991-2003 y Unión libre
Cohorte 2004-2016 y Unión libre

\section{Duración en meses (ref: o-23)}

\begin{tabular}{|c|c|c|c|c|}
\hline $24-47$ & $0,93^{* *}$ & 0,96 & 0,98 & 0,98 \\
\hline $48-71$ & $0,82^{* * *}$ & $0,87^{* * *}$ & $0,93^{* *}$ & $0,93^{*}$ \\
\hline $72-95$ & $0,71^{* * *}$ & $0,77^{* * *}$ & $0,85^{* * *}$ & $0,86^{* * *}$ \\
\hline $96-119$ & $0,67^{* * *}$ & $0,75^{* * *}$ & $0,85^{* * *}$ & $0,85^{* * *}$ \\
\hline $120-143$ & $0,64^{* * *}$ & $0,71^{* * *}$ & $0,82^{* * *}$ & $0,82^{* * *}$ \\
\hline $144-167$ & $0,57^{* * *}$ & $0,66^{* * *}$ & $0,76^{* * *}$ & $0,76^{* * *}$ \\
\hline $168-191$ & $0,52^{* * *}$ & $0,60^{* * *}$ & $0,70^{* * *}$ & $0,70^{* * *}$ \\
\hline $192-215$ & $0,48^{* * *}$ & $0,57^{* * *}$ & $0,67^{* * *}$ & $0,67^{* * *}$ \\
\hline $216-239$ & $0,43^{* * *}$ & $0,51^{* * *}$ & $0,61^{* * *}$ & $0,60^{* * *}$ \\
\hline 240 o más & $0,32^{* * *}$ & $0,39^{* * *}$ & $0,47^{* * *}$ & $0,47^{* * *}$ \\
\hline Constante & $0, \mathrm{OO}^{* * *}$ & $0, \mathrm{OO}^{* * *}$ & $0, \mathrm{OO}^{* * *}$ & $0,00^{* * *}$ \\
\hline AIC & 55549 & 54606,5 & 53806,5 & 53801,5 \\
\hline Número de mujeres & 24823 & 24823 & 24823 & 24823 \\
\hline Número de rupturas & 9608 & 9608 & 9608 & 9608 \\
\hline
\end{tabular}




\section{Conclusiones}

A lo largo del artículo, se ha visto cómo los cambios en los niveles de disolución dan cuenta también de las transformaciones sociales que ha experimentado Colombia en los últimos años, y cómo estas se manifiestan en la duración de los acuerdos conyugales. La evidencia mostrada confirma el aumento de la separación en las uniones formadas entre 1991 y 2003 y, aún más, en las iniciadas a partir de 2004. Con ello se confirma el primer resultado esperado (RE1) de la mayor disolubilidad de las uniones contemporáneas. En la actualidad, según datos de la ENDS 2015 (Profamilia; Ministerio de Salud y Protección Social, 2017), la población entre 15 y 59 años con alguna experiencia de unión registra un nivel de ruptura de la unión de $35 \%$ después de 10 años de haberse iniciado esta, y de $48 \%$ después de 20 años. Sin embargo, estos valores no son homogéneos en los departamentos del país, siendo Boyacá y Nariño los territorios con los niveles más bajos, y Arauca y Chocó los lugares con los valores más altos.

En relación con los determinantes de la disolución, en el presente estudio se abordaron en tres grupos: los asociados con las características de la unión, con las características individuales y de la pareja, y los relacionados con el contexto inmediato. En el primero de estos grupos se confirma el mayor riesgo de separación de las uniones más recientes (RE1), es decir, tanto los resultados descriptivos como los de los modelos multivariados permitirían afirmar que, recuperando la pregunta de la publicación de $L a F M$ con la cual iniciamos el presente artículo, en efecto, "están de moda las separaciones conyugales" (Espejo, 2018), si por ello se hace referencia a un incremento de las rupturas de las uniones. Sin embargo, este patrón se ha venido dando en Colombia desde los setenta (Flórez, 2004; Flórez; Sánchez, 2013; Goldman, 1981; Ruiz-Vallejo, 2020; Zamudio; Rubiano, 1991) y hace parte de una tendencia dada en la mayoría de países de la región (Cabella, 2010; Salinas-Ulloa, 2018; Solís; Ferraris, 2014). De igual manera, hemos comprobado que las rupturas de las uniones libres estarían más de moda que los matrimonios (RE2), incluso después de controlar por el efecto de la cohorte (modelo 4 de la Tabla 5), tal como ocurre en Latinoamérica (Cabella, 2010; Miranda-Ribeiro, 1993; Ojeda, 1986; Pérez-Amador; Ojeda, 2016; Santillán; Street, 2005; Solís; Ferraris, 2014).

Los hallazgos sobre los diferenciales de las características individuales y de la pareja muestran que los menores riesgos de separación los tendrían aquellas que han postergado su unión (RE3), en uniones conformadas por un hombre de 3 a 9 años mayor que la mujer (RE4) y que no tuvieron hijos antes de iniciar la unión (RE6). $\mathrm{El}$ resultado esperado de la fecundidad ( $\mathrm{RE} 7)$ se acepta parcialmente, porque los modelos indican que este efecto opera diferente en función del número y la edad del 
hijo/a más pequeño/a, mientras que la pareja aún está unida. En comparación con no tener hijos, tener entre 1 y 3 no reporta un riesgo diferente de disolución, siempre y cuando el menor de ellos tenga más de 7 años. Cuando aparece un cuarto hijo, sin importar la edad del más pequeño, se observa una reducción del riesgo de separación.

En términos del nivel educativo, la evidencia actual ubicaría a Colombia en un nivel inicial de divorcialidad de acuerdo con la propuesta evolutiva de Goode (1962). En este sentido, se confirma el RE5 sobre el mayor riesgo de disolución de las mujeres con estudios superiores en comparación con el nivel de primaria o menos, tal como ocurre aún en Brasil (Miranda-Ribeiro, 1993) y Chile (Salinas-Ulloa, 2018). Nuestros hallazgos no corroboran la inversión de la escolaridad o la difuminación como un diferencial de la disolución, tal como empieza a observarse en Argentina (Santillán and Street, 2005), Uruguay (Cabella, 2010) o, recientemente, en México (Pérez-Amador; Ojeda, 2016).

Finalmente, en el grupo de diferenciales sobre el contexto inmediato, se encontró evidencia para aceptar el resultado esperado del carácter urbano de las separaciones actuales (RE8), pero no de la difusión del fenómeno en todo el país (RE9), dado que aún regiones como la Atlántica, Central y Pacífica muestran un nivel mayor de ruptura de las uniones, en comparación con la región Oriental. Sin embargo, estos resultados deben ser tomados con cautela, dadas las limitaciones de la fuente, porque tanto la zona como la región de residencia son variables que dan cuenta de la situación actual de la persona y no durante la primera unión. Otra limitación importante del trabajo es la ausencia de información relevante respecto a la primera unión, tal como la condición laboral, la calidad de la relación en términos de la satisfacción con la pareja o con su familia, etc. Por tanto, se recomienda profundizar sobre estos aspectos en encuestas poblacionales que permitan tener en cuenta más elementos involucrados en una situación tan compleja como la ruptura de una unión.

\section{Referencias}

Amato, Paul R. (2000). The Consequences of Divorce for Adults and Children. Journal of Marriage and Family, 62(4), 1269-1287.

Amato, Paul R. (2010). Research on Divorce:Continuing Trends and New Developments. Journal of Marriage and Family, 72(3), 650-666. https://doi.org/10.1111/j.1741-3737.2010.00723.x

Ariza, Marina; Oliveira, Orlandina (2001). Familias en transición y marcos conceptuales en redefinición. Papeles de Población, 7(28), 9-39. 
Beck-Gernsheim, Elizabeth (2003). La reinvención de la familia. En busca de nuevas formas de convivencia. Barcelona: Paidós.

Becker, Gary S.; Landes, Elisabeth M.; Michael, Robert T. (1977). An Economic Analysis of Marital Instability. Journal of Political Economy, 85(6), 1141-1187.

Bernardi, Fabrizio (2006). Análisis de la historia de acontencimientos. Cuadernos Metodológicos No 38. Madrid: CIS.

Bernardi, Fabrizio; Martínez-Pastor, Juan Ignacio (2011). Divorce Risk Factors and their Variations over Time in Spain. Demographic Research, 24(31), 771-800. https://doi. org/10.4054/DemRes.2011.24.31

Bucheli, Marisa; Vigna, Andrés (2005). Un estudio de los determinantes del divorcio en Uruguay. Desarrollo y Sociedad, 1, 1-21. Recuperado de https://revistas.uniandes.edu.co/ doi/pdf/10.13043/dys.56.1

Cabella, Wanda (2008). Dissolução e formação de novas uniões: uma análise demográfica das tendências recentes no Uruguai. Textos NEPO, 56. Recuperado de http://www.nepo. unicamp.br/publicacoes/textos_nepo/textos_nepo_56.pdf

Cabella, Wanda (2010). Los determinantes de la ruptura de la primera unión en el Uruguay: un análisis a partir de dos encuestas retrospectivas. Revista Latinoamericana de Poblacion, 7, 31-56.

Castro-Martín, Teresa (2002). Consensual Unions in Latin America: Persistence of a Dual Nuptiality System. Journal of Comparative Family Studies, 33(1), 35-55.

Cherlin, Andrew J. (2010). The Marriage-Go-Round. The State of Marriage and the Family in America Today. New York: Vintage.

Colombia Diversa (2017). Familias bajo sospecha. La batalla por la igualdad de las parejas del mismo sexo en Colombia. Bogotá: Colombia Diversa.

Coppola, Lucia; Di Cesare, Mariachiara (2008). How fertility and union stability interact in shaping new family patterns in Italy and Spain. Demographic Research, 18, 117-44. https:// doi.org/10.4054/DemRes.2008.18.4

Corporación Centro Regional de Población; Ministerio de Salud de Colombia; Institute for Resource Development (1988). Encuesta de Prevalencia Demografia y Salud 1986. Bogotá: CCRC-Ministerio de Salud-IRD. Recuperado de https://www.dhsprogram.com/pubs/ pdf/FR8/FR8.pdf

Departamento Administrativo Nacional de Estadística (2020). Censo Nacional de Poblacióny Vivienda 2018. Recuperado de http://systema59.dane.gov.co/bincol/RpWebEngine.exe/ Portal?BASE=CNPVBASE4V2\&lang=esp 
Ermisch, John (2003). Does a 'Teen-Birth' Have Longer-Term Impacts on the Mother? Suggestive Evidence From the British Household Panel Study. Working Papers of the Institute for Social and Economic Research, 32. Recuperado de https://www.iser.essex.ac.uk/files/ iser_working_papers/2003-32.pdf.

Escobar, Modesto; Fernández, Enrique; Bernardi, Fabrizio (2009). Análisis de datos con Stata. Cuadernos Metodológicos 45. Madrid: CIS.

Espejo, Germán (29 de agosto de 2018). Al día se presentan 60 divorcios en Colombia. La FM. Recuperado de https://www.lafm.com.co/colombia/al-dia-se-presentan-60-divorciosen-colombia

Esping-Andersen, Gøsta; Billari, Francesco C. (2015). Re-theorizing Family Demographics. Population and Development Review, 41(1), 1-31. https://doi.org/10.1111/j.17284457.2015.00024.x

Esteve, Albert; Lesthaeghe, Ron; Lopez-Gay, Antonio (2012). The Latin American Cohabitation Boom, 1970 2007. Population and Development Review, 38(1), 55-81.

Esteve, Albert; López, Luis Ángel; McCaa, Robert (2013). The Educational Homogamy Gap Between Married and Cohabiting Couples in Latin America. Population Research and Policy Review, 32(1), 81-102. https://doi.org/10.1007/s11113-012-9263-4

Fernández, Mariana (2010). Estudio sobre las trayectorias conyugales de las mujeres del Gran Montevideo. México: Flacso.

Flórez, Carmen E. (2000). Las transformaciones sociodemográficas en Colombia durante el siglo $X X$. Bogotá: Banco de la República.

Flórez, Carmen E. (2004). La transformación de los hogares: una visión de largo plazo. Coyuntura social, 30, 23-49.

Flórez, Carmen E.; Sánchez, Lina M. (2013). Fecundidad y familia en Colombia: ¿hacia una segunda transición demográfica? Estudios a profundidad basados en las Encuestas Nacionales de Demografia y Salud, ENDS 1990-2010. Serie. Bogotá: Profamilia.

García, Brígida; Rojas, Olga (2004). Las uniones conyugales en América Latina:transformaciones en un marco de desigualdad social y de género. Notas de población, 78, 65-96.

Giddens, Anthony (1997). La transformación de la intimidad: sexualidad, amory erotismo en las sociedades modernas. Madrid: Cátedra.

Goldman, Noreen (1981). Dissolution of First Unions in Colombia, Panama, and Peru. Demography, 18(4), 659-679. 
Goldscheider, Frances; Bernhardt, Eva; Lappegård, Trude (2015). The Gender Revolution: A Framework for Understanding Changing Family and Demographic Behavior. Population and Development Review, 41(2), 207-239. https://doi.org/10.1111/j.1728-4457.2015.00045.x

Gómez, Maribel (2011). ¿Separación, divorcio o viudez? Cambios en la disolución de uniones conyugales de mujeres mexicanas. En Parejas conyugales en transformación (pp. 167-210), editado por Julieta Quilodrán. México: El Colegio de México.

Goode, W. J. (1962). Marital Satisfaction and Instability. A Cross-Cultural Class Analysis of Divorce Rates. En Class, Status and Power. Social Stratification in Comparative Perspective (pp. 377-387), editado por Reinhard Bendix; Seymour Lipset. New York: The Free Press.

Härkönen, Juho (2014). Divorce: Trends, Patterns, Causes, and Consequences. En The Wiley Blackwell Companion to the Sociology of Families (pp. 303-322), editado por Judith Treas; Jacqueline Scott; Martin Richards. Oxford: John Wiley \& Sons, Inc.

Härkönen, Juho; Dronkers, Jaap (2006). Stability and Change in the Educational Gradient of Divorce. A Comparison of Seventeen Countries. European Sociological Review, 22(5), 501517. https://doi.org/10.1093/esr/jcl011

Jalovaara, Marika; Kulu, Hill (julio, 2018). Separation Risk over Union Duration: An Immediate Itch? European Sociological Review, 1-15. https://doi.org/10.1093/esr/jcy017

Kalmijn, Matthijs (1998). Intermarriage and Homogamy: Causes, Patterns, Trends. Annual Review of Sociology, 24(1), 395-421. https://doi.org/10.1146/annurev.soc.24.1.395

Kuperberg, Arielle (2014). Age at Coresidence, Premarital Cohabitation, and Marriage Dissolution: 1985-2009. Journal of Marriage and Family, 76(2), 352-369. https://doi. org/10.1111/jomf.12092

Lehrer, Evelyn L. (2008). Age at Marriage and Marital Instability: Revisiting the BeckerLandes-Michael Hypothesis. Journal of Population Economics, 21(2), 463-484. https://doi. org/10.1007/s00148-006-0092-9

Lesthaeghe, Ron (2010). The Unfolding Story of Transition. Population and Development Review, 36(2), 211-251. https://doi.org/10.1111/j.1728-4457.2010.00328.x

Lillard, Lee A.; Waite, Linda J. (1993). A Joint Model of Marital Childbearing and Marital Disruption. Demography, 30(4), 653-681.

Lyngstad, Torkild Hovde; Jalovaara, Marika (2010). A Review of the Antecedents of Union Dissolution. Demographic Research, 23(2), 257-292. https://doi.org/10.4054/ DemRes.2010.23.10

Miranda-Ribeiro, Paula (1993). Começar de novo: um estudo comparativo do descasamento e recasamento. [Tesis de maestría]. Universidade Federal de Minas Gerais, Facultad de Ciencias Económicas, Maestría en Demografía. 
Mortelmans, Dimitri (2020). Divorce in Europe. New Insights in Trends, Causes and Consequences of Relation Break-ups. Cham: Springer Open. https://doi.org/10.1007/978-1-4613-4236-6

Ojeda, Norma (1986). La separación y el divorcio en México vistos desde la perspectiva demográfica. Estudios Demográficos y Desarrollo Urbano, 1(2), 227-265.

Ojeda, Norma; González, Eduardo (2008). Divorcio y separación conyugal en México en los albores del siglo XXI. Revista Mexicana de Sociología, 70(1), 111-145.

Pérez-Amador, Julieta (2008). Análisis multiestado multivariado de la formación y disolución de las parejas conyugales en México. Estudios Demográficos y Urbanos, 23(3), 481-511.

Pérez-Amador, Julieta; Ojeda, Norma (2016). Una nueva mirada a los factores predictivos de la disolución conyugal voluntaria en México. En Generaciones, cursos de vida y desigualdad social en México (pp. 223-254), editado por Marie-Laure Coubès; Patricio Solís; María Eugenia Zavala de Cosío. México: El Colegio de México/El Colegio de la Frontera Norte.

Profamilia; Ministerio de Salud y Protección Social (2017). Encuesta Nacional de Demografíay Salud 2015 (Tomo I). Bogotá: Profamilia/MSPS.

Raley, Kelly; Sweeney, Megan (2020). Divorce, Repartnering, and Stepfamilies: A Decade in Review. Journal of Marriage and Family, 82(1), 81-99. https://doi.org/10.1111/jomf.12651

Ruiz-Vallejo, Fernando (2018). La separación conyugal en Colombia, 1951-2015: geografías y biografias en clave de género. Barcelona: Universitat Autònoma de Barcelona.

Ruiz-Vallejo, Fernando (2020). La separación conyugal en los censos y en las encuestas de demografía y salud en Colombia, 1951-2015. Sociedad y Economía, 39, 155-182. https:// doi.org/10.25100/sye.v0i39.7913

Salinas-Ulloa, Viviana (2018). Hacia la medición del riesgo de disolución del matrimonio en Chile. Estudios Demográficos y Urbanos, 33(3), 769-798. https://doi.org/10.24201/edu. v33i3.1720

Salvini, Silvana; Vignoli Daniele (2011). Things Change: Women's and Men's Marital Disruption Dynamics in Italy During a Time of Social Transformations, 1970-2003. Demographic Research, 24(5), 145-174. https://doi.org/10.4054/DemRes.2011.24.5

San Juan, Victoria; Esteve, Albert (2019). Amores imposibles: la brecha entre universitarios y el resto de grupos educativos en los mercados matrimoniales de América Latina, 19702010. Notas de Población, 108, 11-36.

Santillán, María Marta; Street, María Constanza (2005). La primera unión y la ruptura conyugal en el curso de vida femenino. Algunas evidencias a partir de la ESF. En Trayectorias nupciales, familias ocultas, Buenos Aires, entresiglos (pp.119-168), editado por Susana Torrado. Buenos Aires: CIEPP/Miño y Dávila. 
Solís, Patricio; Ferraris, Sandra (2014). Nuevo siglo, ¿nuevas pautas de formación y disolución de uniones? En Los mexicanos. Un balance del cambio demográfico (pp. 269-305), editado por Cecilia Rabell. México: FCE.

Solís, Patricio; Puga, Ismael (2009). Los nuevos senderos de la nupcialidad: cambios en los patrones de formación y disolución de las primeras uniones. En Tramas familiares en el México contemporáneo. Una perspectiva sociodemográfica (pp.179-198), editado por Cecilia Rabell. México: IIS-UNAM/El Colegio de México.

StataCorp (2011). Stata Statistical Software: Release 12. College Station. Texas: StataCorp LP.

Torrado, Helí Abel (2016). Derecho de familia. Matrimonio, filiación y divorcio (2. ${ }^{\mathrm{a}}$ ed.). Bogotá: Universidad Sergio Arboleda.

Van de Kaa, Dick (enero, 2002). The Idea of a Second Demographic Transition in Industrialized Countries. Trabajo presentado en Sixth Welfare Policy Seminar, National Institute of Population and Social Security, Tokyo.

Vignoli, Daniele; Ferro, Irene (2009). Rising Marital Disruption in Italy and its Correlates. Demographic Research, 20(4), 11-36. https://doi.org/10.4054/DemRes.2009.20.4

Waite, Linda J.; Lillard, Lee A. (1991). Children and Marital Disruption. American Journal of Sociology, 96(4), 930-953.

Zamudio, Lucero; Rubiano, Norma (1991). Las separaciones conyugales en Colombia. Bogotá: Universidad Externado de Colombia. 